\title{
LETTER TO THE EDITORS
}

\section{Case Letter}

Dear Editor,

\section{Chemotherapy-induced alopecia: A novel observation}

Chemotherapy-induced alopecia is a psychologically distressing consequence of some cancer treatment. Incidence is estimated at $65 \%$ with 'classic' anticancer drugs and $14.7 \%$ with new targeted therapies. ${ }^{1}$ Hair-shaft shedding can appear within 2 weeks (anagen effluvium), or less often, months after the beginning of therapy (telogen effluvium). Although permanent alopecia has been reported in up to $20 \%$ of cases, chemotherapy-induced alopecia is usually reversible, even if texture and colour persist. ${ }^{2,3}$
We describe a novel trichoscopic pattern of chemotherapy-induced alopecia. We observed two patients with chemotherapy-induced alopecia whose hair regrew 'black and white' after three cycles of 5-fluorouracil, epirubicin and cyclophosphamide and 12 cycles of taxanes for breast cancer. Hairs that emerged from follicular ostia appeared non-pigmented, but within a few millimetres of length they became pigmented, appearing bicolored (white at the tips and pigmented at the base; Figs 1,2).

Chemotherapy affects hair pigmentation with different mechanisms. It causes oxidative damage to the hair follicle pigmentary unit, and induces a complex melanocyte response. ${ }^{4}$ Moreover, enzymatically determined intrafollicular synthesis of eumelanin (black) and pheomelanin (red) may also be affected. ${ }^{5}$

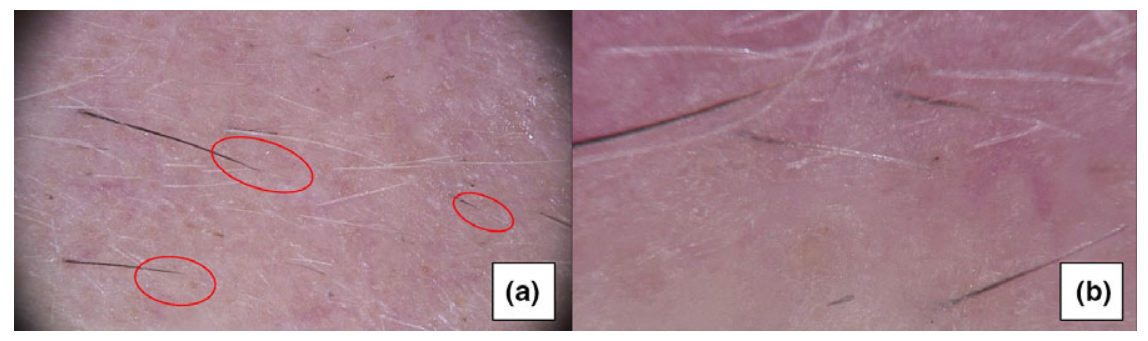

Figure 1 Trichoscopy of the first patient shows several 'black and white' hairs.

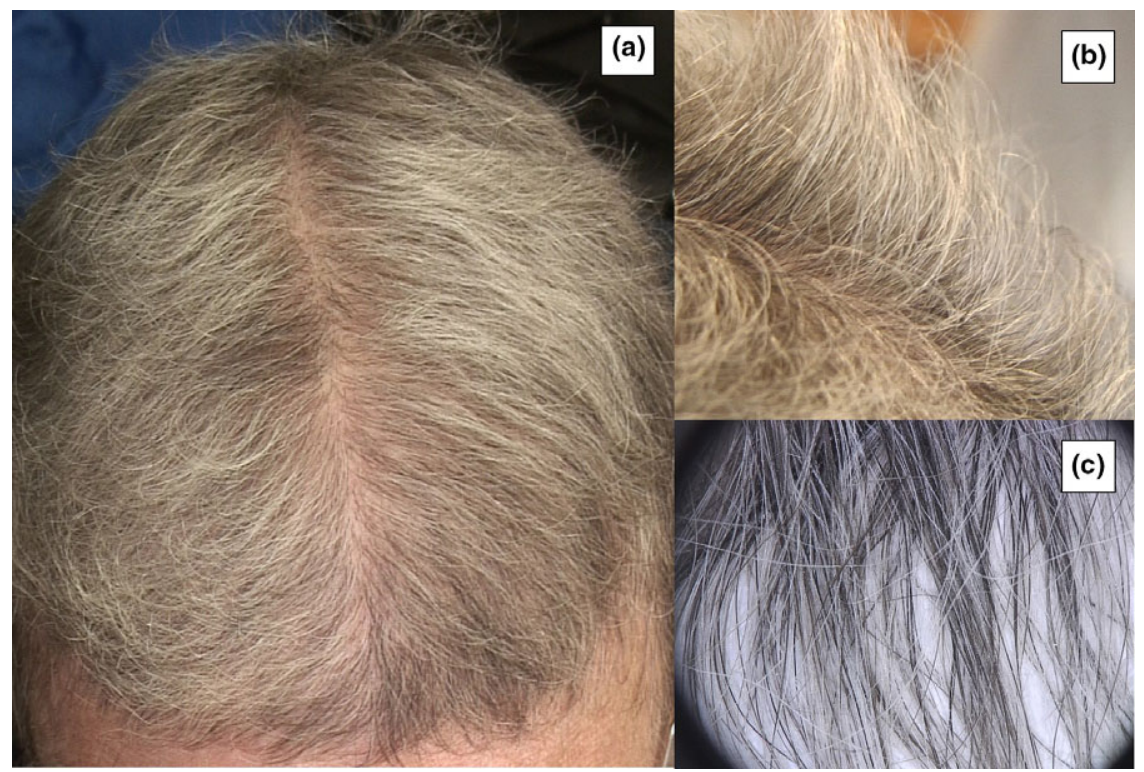

Figure 2 (a, b) Clinical and (c) trichoscopic images of the second patient: the hair shaft appears white at the tips and pigmented at the base.

Conflict of interest: none. 
We hypothesize that our observation is the result of a reactivation of follicular melanocytes that were damaged but not destroyed. It is conceivable that this reflects the end of a taxane-associated blockade of microtubules that leads to a paralysis of melanosome transport. ${ }^{6}$ A further possible mechanism could be the migration of amelanotic melanocytes residing in the root sheath, which were recalled and activated by morphogenetic signals generated by the follicular niche. This last hypothesis is supported by the fact that both patients had white hair prior to chemotherapy. It is possible that recovery after chemotherapy could activate senescent pigmentary units.

The phenomenon we observed is not exclusive to chemotherapy-induced alopecia as white tips are a normal occurrence on nasal hairs and in regrowth after alopecia areata. Chemotherapy-induced alopecia differs to alopecia areata, which is characterized by the presence of vellus-like hair during the recovering phase. These hairs are destined to complete the hair-cycle, to fall out, and then to be replaced by pigmented terminal hair. ${ }^{7}$ Chemotherapy-induced alopecia, on the other hand, does not necessarily need a whole hair cycle to reestablish pigmentation.

A longer observation time is required to determine how long these pigmentary modifications last and further studies are necessary for a better understanding of this process.

\footnotetext{
Alfredo Rossi $^{1}$ | Maria Caterina Fortuna ${ }^{1}$ | Gemma Caro $^{1}$ iD | Flavia Pigliacelli ${ }^{1}$ | Andrea D'Arino $^{1}$ iD | Marta Carlesimo ${ }^{2}$
}

${ }^{1}$ Department of Internal Medicine and Medical Specialties,
and ${ }^{2}$ Department of Dermatology, II School, Sapienza
University of Rome, Rome, Italy

\section{REFERENCES}

1. Belum VR, Marulanda K, Ensslin C et al. Alopecia in patients treated with molecularly targeted anticancer therapies. Ann. Oncol. 2015; 26: 2496-502.

2. Tosti A, Piraccini BM, Vincenzi C et al. Permanent alopecia after busulfan chemotherapy. Br. J. Dermatol. 2005; 152: 10568.

3. Paus R, Haslam IS, Sharov AA et al. Pathobiology of chemotherapy-induced hair loss. Lancet Oncol. 2013; 14: e50-9.

4. Wood JM, Decker H, Hartmann H et al. Senile hair graying: H2O2-mediated oxidative stress affects human hair color by blunting methionine sulfoxide repair. FASEB J. 2009; 23: 206575 .

5. Tobin DJ. The cell biology of human hair follicle pigmentation. Pigment Cell Melanoma Res. 2011; 24: 75-88.

6. Sibaud V, Lebœuf NR, Roche $\mathrm{H}$ et al. Dermatological adverse events with taxane chemotherapy. Eur. J. Dermatol. 2016; 26: $427-43$.

7. Bodó E, Tobin DJ, Kamenisch Y et al. Dissecting the impact of chemotherapy on the human hair follicle: a pragmatic in vitro assay for studying the pathogenesis and potential management of hair follicle dystrophy. Am. J. Pathol. 2007; 171: 1153-67. 